Journal of Animal and Veterinary Advances 11 (14): 2398-2402, 2012

ISSN: $1680-5593$

(C) Medwell Journals, 2012

\title{
An Improved Reverse Transcription Loop-Mediated Isothermal Amplification Assay for Sensitive and Specific Detection of Infectious Bronchitis Virus
}

\author{
${ }^{1}$ Hongbin Luo, ${ }^{2}$ Daozhong Zhu, ${ }^{1}$ Chunyi Xue, ${ }^{3}$ Jianping Qin, ${ }^{3}$ Feng Chen and ${ }^{1}$ Yongchang Cao \\ ${ }^{1}$ State Key Laboratory of Biocontrol, School of Life Sciences, \\ Sun Yat-sen University, 510006, Guangzhou, P.R. China \\ ${ }^{2}$ Technical Center, Guangdong Entry-Exit Inspection and Quarantine Bureau, \\ 510623 Guangzhou, P.R. China \\ ${ }^{3}$ Guangdong Wen's Food Co. Ltd., 527400 Xinxing, Guangdong Province, P.R. China
}

\begin{abstract}
A highly conserved region of $1 a$ gene of Infectious Bronchitis Virus (IBV) was chosen to design the Reverse Transcription Loop-Mediated Isothermal Amplification (RT-LAMP) degenerate primers. The developed RT-LAMP assay is a highly sensitive, specific, rapid method to detect IBV in allantoid and tissue. Optimal temperature was around $62^{\circ} \mathrm{C}$ and duration was $45 \mathrm{~min}$. Sensitivity analysis showed that RT-LAMP assay was 10 fold more sensitive than RT-PCR. Specificity analysis showed that 3 IBV strains omitted by the previous reported RT-LAMP assay could be detected by the improved RT-LAMP assay. In field trials, the improved RT-LAMP assay obtained $98.4 \%$ sensitivity in 65 clinical samples while $95.4 \%$ of RT-PCR and $93.8 \%$ of the previous reported RT-LAMP which indicates it is a powerful tool in the practical application.
\end{abstract}

$\underline{\text { Key words: Infectious bronchitis virus, loop-mediated isothermal amplification, IBV strains, RT-LAMP }}$

\section{INTRODUCTION}

Infectious Bronchitis (IB), an economically important disease to poultry industry is caused by Infectious Bronchitis Virus (IBV) which is a gammacoronavirus in the family Coronaviridae, the order Nidovirales. IBV, a highly infectious pathogen of chicken, not only causes upper respiratory disease but also replicates in kidney and oviduct.

IBV is an enveloped, positive-stranded RNA virus and the $27 \mathrm{~Kb}$ IBV genome contains nine functional genes, four of which encode structural proteins: Envelope (E) glycoprotein, integral Membrane (M) glycoprotein, phosphorylated Nucleocapsid (N) protein and Spike (S) glycoprotein (Boursnell et al., 1987; Lai and Cavanagh, 1997).

Though commercial chickens have been vaccinated, IB outbreaks still happen in many countries. Therefore, rapid, sensitive, specific and simple diagnostic methods for detecting IBV are in a great need to develop for controlling the disease. Conventional methods for detection of IBV include virus isolation, cell culture immunoassays and molecular assays that detect the viral RNA (Cavanagh et al., 1992; Swayne, 1998; Hosseini et al., 2011). However, all of these conventional methods are considered to be time-consuming with the requirement of specialized equipment and unsuitable for rapid and routine detection of IBV. A novel Nucleic Acid
Amplification Method termed Reverse Transcription Loop-Mediated Isothermal Amplification (RT-LAMP) was developed. It relies on the principle of a strand displacement reaction forming a stem-loop structure so that it amplifies a target gene with high efficiency, specificity and sensitivity which only needs simple device such as water bath or heat block for thermal change due to its isothermal reaction (Nagamine et al., 2002; Notomi et al., 2000).

The amplified products can be analyzed by agarose gel electrophoresis and the white precipitate or a visible color change with the addition of SYBR Green I to the amplified product which is visible to naked eyes (Mori et al., 2001).

Chen et al. (2010) reported an RT-LAMP assay targeting the nucleocapsid phosphoprotein gene (pre-NRT-LAMP) that was capable of detecting a wide collection of IBV clinical tissue samples ( 88 blood, 62 kidney and 37 lung) but they did not demonstrate its application in field clinical samples. The routine surveillance of IBV revealed that pre-N-RT-LAMP produced serious false negative results. In this study, researchers have developed a new 1 step RT-LAMP assay with degenerate primers targeting the $1 a$ gene for detection of IBV. The RT-LAMP assay showed improved sensitivity and specificity over the RT-LAMP assay reported by Chen et al. (2010).

Corresponding Author: Yongchang Cao, School of Life Sciences, Sun Yat-sen University, 510006 Guangzhou, P.R. China 


\section{MATERIALS AND METHODS}

IBV isolates, control viruses and field clinical samples: Twenty IBV isolates were obtained from China between 2009 and 2010. Virus was propagated in 9-11 days old embryonated Special Pathogen Free (SPF) chicken eggs and identified by RT-PCR of the $\mathrm{N}$ protein gene. Moreover, several blind passages were performed until the dwarfing and death of embryos were observed between 2 and 7 days after inoculation. Standard IBV vaccine strain H52 (Merial Animal Health) was used as the reference virus to explore the optimal reaction conditions and detection sensitivity of RT-LAMP. The control viruses included Newcastle Disease Virus (NDV) provided by China Institute of Veterinary Drug Control, Infectious Bursal Disease Virus (IBDV), Avian Leukosis Virus (ALV), Infectious Laryngotracheitis Virus (ILTV) and the Low Pathogenicity-Avian Infiuenza Virus (LP-AIV) H9N2 which were isolated and provided by Guangdong Wen's Food Co. Ltd.

The field clinical samples were obtained from 80 chicken flocks that were suspected to be infected with IBV from 2009-2010. All samples were placed in isotonic Phosphate-Buffered Saline (PBS), pH 7.4, containing antibiotics and stored at $-80^{\circ} \mathrm{C}$ until total RNA was extracted.

Total RNA extraction: RNA was extracted from allantoic fluids using the Axy Prep ${ }^{\mathrm{TM}}$ Body Fluid Viral DNA/RNA Miniprep kit (AXYGEN), according to the manufacturer's instructions. Total RNA was stored at $-80^{\circ} \mathrm{C}$ until use.

Primers and one-step RT-LAMP reaction: Based on the alignment of IBV strain sequences published in GenBank, a highly conserved region of the $1 a$ gene was chosen to design the RT-LAMP primers with degenerate oligonucleotides. Four primers, F3, B3, FIP and BIP were designed using Primer Explorer Version 3 (http://primer explorer.jp/lamp3.0.0/index.html) (Table 1). The reaction mixture contained $2 \mathrm{M}$ each of the inner primers FIP and $\mathrm{BIP}, 0.5 \mathrm{M}$ each of the outer primers $\mathrm{F} 3$ and B3, $1.4 \mathrm{mM}$ dNTP mix (TaKaRa), 0.6 Mbetaine (Sigma), $5 \mathrm{mMMgSO}_{4}$, $8 \mathrm{U}$ of Bst DNA polymerase (large fragment; New England

Table 1: The RT-PCR and RT-LAMP primer sets. The degenerate oligonucleotide indicated in italic type, $\mathrm{R}=\mathrm{G}+\mathrm{A}, \mathrm{Y}=\mathrm{C}+\mathrm{T}$. The restriction sites of Eco RV were added between the B1c and B2, indicated in bold type

\begin{tabular}{ll}
\hline Primer name & Sequence $\left(5^{\prime}-3^{\prime}\right)$ \\
\hline F3 & CCTCTGGTTCATCTAGCGGT \\
B3 & GGGTTGTTYGGCACTACC \\
FIP & GAGGTGACCCCGTGTTTCACGATATCGGGTGT \\
& GTGGAAGTAGC \\
BIP & AGGGCTTTTGAGCCTAGCGTGGATCCCTACAA \\
& CCGTCGTATAGC \\
\hline
\end{tabular}

Biolabs), $1 \times$ the supplied Bst DNA polymerase buffer, $0.125 \mathrm{U}$ of AMV reverse transcriptase (TaKaRa) and $2 \mu \mathrm{L}$ of template RNA in a final volume of $25 \mu \mathrm{L}$. The RT-LAMP reaction mixtures were incubated at $65^{\circ} \mathrm{C}$ for $30 \mathrm{~min}$ and finally heat inactivated at $80^{\circ} \mathrm{C}$ for $5 \mathrm{~min}$ to terminate the reaction.

Analysis of DNA products from the RT-LAMP assay: The amplified DNA products from the RT-LAMP reaction mixture were analyzed in three ways:

- Agarose gel electrophoresis: a $5 \mu \mathrm{L}$ aliquot of the RT-LAMP reaction mixture was separated on a $2 \%$ agarose gel stained with ethidium bromide

- Visual observation of white precipitates: using this method, the result can be judged without opening the tubes and the volatilization of RT-LAMP product can be avoided

- Colorimetric assay: about $1 \mu \mathrm{L}$ of SYBR Green I dye (Beijing Dingguo Biotechnology) was added to the tube after the reaction and positive reaction mixtures changed color from yellow to green

RT-PCR: Primers of F3 and B3 were chosen to RT-PCR. According to the manufacturer's recommendations, 1 step RT-PCR reaction mixture was set up as follows: $2 \mu \mathrm{L}$ of PrimeScript 1 Step Enzyme Mix, $25 \mu \mathrm{L}$ of $2 \times 1$ Step Buffer, $1 \mu \mathrm{L}$ of $20 \mu \mathrm{mol} \mathrm{L}^{-1}$ of each of sense primer and anti-sense primer, $5 \mu \mathrm{L}$ of template RNA, $21 \mu \mathrm{L}$ of RNase Free $\mathrm{ddH}_{2} \mathrm{O}$. The PCR conditions for amplification were $50^{\circ} \mathrm{C}$ for $30 \mathrm{~min}$, $94^{\circ} \mathrm{C}$ for $5 \mathrm{~min}, 30 \mathrm{cycles}$ of $94^{\circ} \mathrm{C}$ for $30 \mathrm{sec}, 52^{\circ} \mathrm{C}$ for $30 \mathrm{sec}$ and $72^{\circ} \mathrm{C}$ for $30 \mathrm{sec}$, followed by $72^{\circ} \mathrm{C}$ for $10 \mathrm{~min}$. The products were analyzed on $2 \%$ agarose gel.

Sensitivity and specificity of the RT-LAMP assay: The sensitivity of the RT-LAMP assay was determined using 10 -fold serial dilutions of RNA template. The specificity of the RT-LAMP assay was determined using 20 IBV isolates as positive controls and 5 other avian viruses as negative controls (i.e., ALV, H9N2 AIV, ILV, NDV and IBDV). All of these amplification reactions were compared to 1 step RT-PCR assay.

\section{RESULTS}

Optimal temperature and reaction duration of RT-LAMP reaction: Temperature and reaction duration were 2 parameters critical in RT-LAMP reaction. To determine the optimal reaction temperature, the RT-LAMP reactions were carried out for $30 \mathrm{~min}$ at $61-65^{\circ} \mathrm{C}$, respectively. The intensities of DNAs from the reactions at $61-63^{\circ} \mathrm{C}$ were apparently higher than those at other temperatures and 


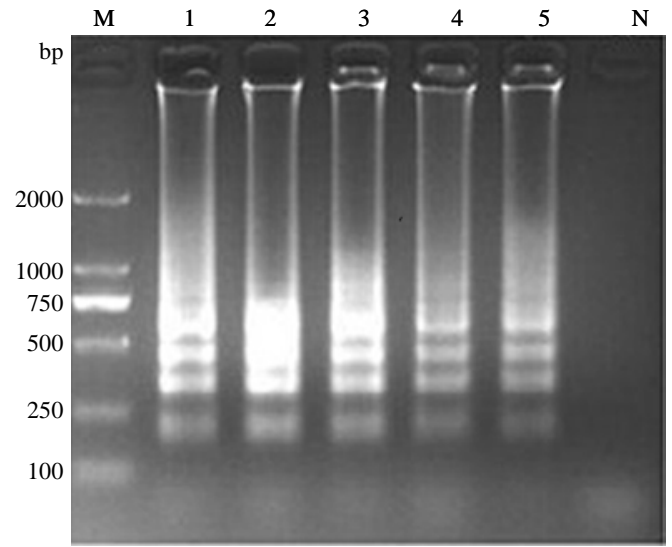

Fig. 1: DNA products from reactions optimizing the temperature of RT-LAMP reaction. Lane $\mathrm{M}=$ DL2000 DNA Marker; Lane N = Negative control; Lane 1 5 $=$ The reaction temperatures, $61 \sim 65^{\circ} \mathrm{C}$

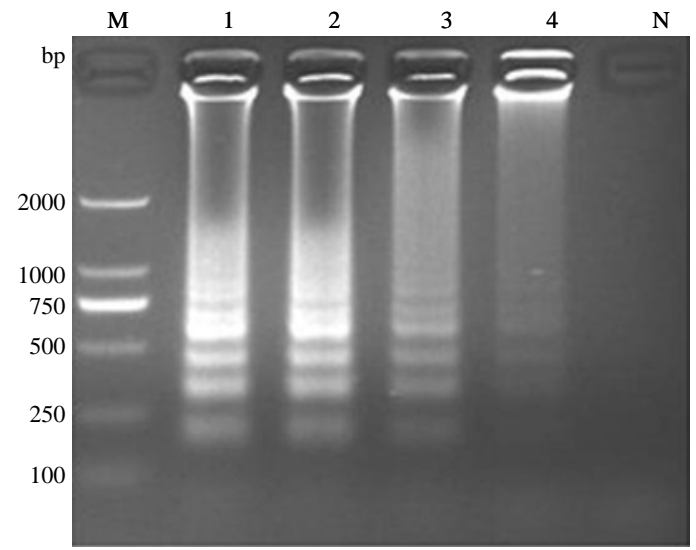

Fig. 2: DNA products from reactions optimizing the reaction duration of RT-LAMP reaction. Lane $\mathrm{M}=$ DL2000 DNA Marker; Lane $\mathrm{N}=$ Negative control; $1-4,60,45,30$ and $15 \mathrm{~min}$ of the reaction duration (min)

$62^{\circ} \mathrm{C}$ was considered as the optimal temperature (Fig. 1). As for optimizing reaction time, the total reaction mixture was incubated at $62^{\circ} \mathrm{C}$ for $60,45,30$ and $15 \mathrm{~min}$ and $45 \mathrm{~min}$ was considered as the optimal reaction duration for the RT-LAMP reaction for detecting IBV (Fig. 2).

Sensitivity and specificity of RT-LAMP: To ascertain the sensitivity of the RT-LAMP assay, 10-fold serial dilutions of RNA templates extracted from IBV H120 were tested and compared to that of the conventional RT-PCR assay (Fig. 3). The result showed that the RT-LAMP assay had a detection limit of $10^{6}$-fold dilution whereas RT-PCR was able to detect at $10^{5}$-fold dilution. Therefore, the sensitivity of 1 step RT-LAMP assay for detecting IBV is
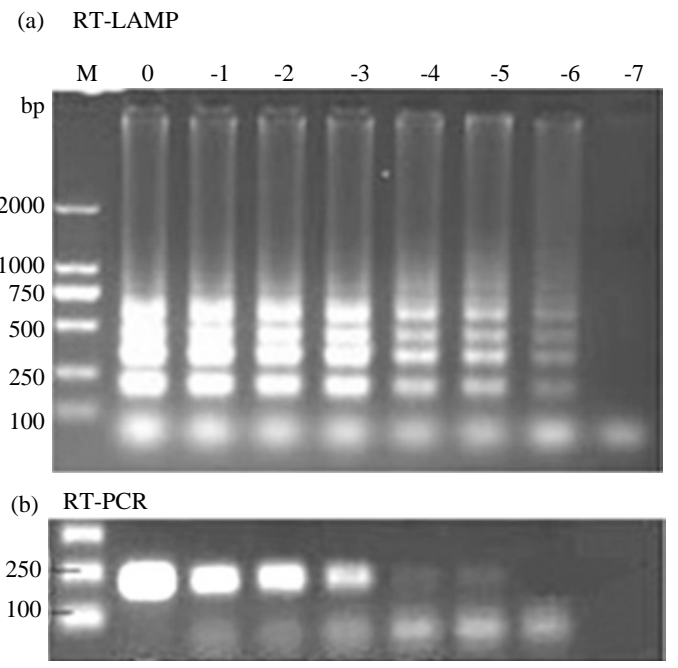

Fig. 3: Comparison of sensitivity of a) RT-LAMP and b) RT-PCR. Lane M = DL2000 DNA Marker; Lane $0 \sim-7(\log 10)$, the amplification product with the 10 fold serial dilution of RNA templates. The RT-PCR product length was about $186 \mathrm{bp}$

Table 2: Analy sis results of field samples

\begin{tabular}{lc}
\hline Clinical samples & Positive result/No. of samples \\
\hline Virus isolation & $65 / 80$ \\
RT-LAMP & $64 / 80$ \\
Pre-N-RT-LAMP & $62 / 80$ \\
RT-PCR & $61 / 80$ \\
\hline
\end{tabular}

about 10 times higher than that of RT-PCR assay. The specificity of the RT-LAMP assay was determined with 20 IBV isolates and 5 other avian viruses. The results of all $20 \mathrm{IBV}$ isolates were positive while that of all 5 other avian viruses were negative. This demonstrated that 1 step RT-LAMP assay was specific with no cross-reaction with other avian viruses.

Application of 1 step RT-LAMP assay to clinical samples: Researchers collected 80 clinical samples and all these samples were subjected to virological diagnosis, of which 65 clinical samples were positive for virus isolation and 15 were negative (Table 2). All of the negative samples were shown to be negative by RT-LAMP, pre-N-RT-LAMP and RT-PCR assays. As for the RT-PCR and pre-N-RT-LAMP assays, four and three positive samples were negative resulting in a sensitivity of 93.8 and $95.4 \%$, respectively. With 1 step RT-LAMP assay, only one positive sample was negative, resulting in a sensitivity of $98.4 \%$.

\section{DISCUSSION}

LAMP is a novel nucleic acid amplification method which has already been applied for detection of several 
avian virus such as Newcastle disease virus (Pham et al., 2005), infectious bursal disease virus (Xue et al., 2009), infiuenza A (H5N1) virus (Imai et al., 2006), infiuenza A (H9) virus (Chen et al., 2008) and so on.

In this study, researchers have developed an improved, sensitive, specific and accelerated 1 step RT-LAMP assay with degenerate primers for detection of IBV isolates and clinical samples. The advantages and potential application of this technique has been examined here which only need a poorly appointed laboratory and can be performed $<1 \mathrm{~h}$. Furthermore, the amplified DNA products can be visualized by colorimetric methods, making it an ideal tool for use in the field to provide instant results. The 1 step RT-LAMP assay is specific and sensitive. The specificity of 1 step RT-LAMP assay for IBV was demonstrated by assaying IBV isolates and five other avian viruses (i.e., ALV, H9N2 AIV, ILV, NDV and IBDV). The results showed that there was no crossreaction with any of the five tested avian viruses. It is also important whether it was applicable to diverse IBV isolates. Four primers targeting the $1 a$ gene was employed because it is highly conserved. Furthermore, at some variable sites inside the primers, researchers used the degenerate oligonucleotides compatible with as many strains as possible. In order to address this issue, 20 different isolates were tested positive. Thus, the RTLAMP assay is widely applicable for diverse IBV strains. The sensitivity of 1 step RT-LAMP assay was tested in two experiments. When researchers used the same templates, 1 step RT-LAMP assay had about 10 fold higher sensitivity than the RT-PCR assay.

In another experiment when 80 clinical samples were used, 1 step RT-LAMP assay had 98.4\% sensitivity while the pre-N-RT-LAMP and RT-PCR had only 95.4 and 93.8\% sensitivity, respectively. Researchers found that the 1 step RT-LAMP missed one positive sample, the reasons for which are still unclear. By sequencing the $1 a$ gene of IBV isolated from the missed sample, it is out of the question that mutations might cause mismatches between the viral RNA and primers. One possible reason is that the amount of IBV in the missed sample was so low that the amount of extracted viral RNA was not enough to trigger the LAMP reaction. To solve the problem, different extraction protocols and kits should be tried for improving the extraction efficacy.

Five isolates were not detected by the pre-N-RTLAMP assay and researchers found the reason is the mismatches between the viral RNA and primers.

\section{CONCLUSION}

The study presented an improved, sensitive, specific, 1 step and accelerated RT-LAMP diagnostic technique to detect IBV. The RT-LAMP assay is feasible for use in less well-equipped laboratories and in the field. It can be used as a substitute for virological diagnosis in routine screening of poultry for rapid diagnosis of IBV.

\section{ACKNOWLEDGEMENTS}

This research was supported by the grants from State Public Industry Scientific Research Programs (2007GYJ019) and Science Technology Strategic Plan (2009B020307008, 2009B020201008) of Guangdong, People's Republic of China.

\section{REFERENCES}

Boursnell, M.E.G., T.D.K. Brown, I.J. Foulds, P.F. Green, F.M. Tomley and M.M. Binns, 1987. Completion of the sequence of the genome of the Coronavirus avian infectious bronchitis virus. J. Gen. Virol., 68: $55-77$.

Cavanagh, D., P.J. Davis, J.K. Cook, D. Li, A. Kant and G. Koch, 1992. Location of the amino acid differences in the S1 spike glycoprotein. Avian Pathol., 21: 33-43.

Chen, H.T., J. Zhang, D.H. Sun, L.N. Ma, X.T. Liu, X.P. Cai and Y.S. Liu, 2008. Development of reverse transcription loop-mediated isothermal amplification for rapid detection of $\mathrm{H} 9$ avian influenza virus. J. Virol. Methods, 151: 200-203.

Chen, H.T., J. Zhang, Y.P. Ma, L.N. Ma and Y.Z. Ding et al., 2010. Reverse transcription loopmediated isothermal amplification for the rapid detection of infectious bronchitis virus in infected chicken tissues. Mol. Cell. Probes, 24: 104-106.

Hosseini, S., J. Arshami and M.E. Torshizi, 2011. Viral antibody titer and leukocyte subset responses to graded copper and zinc in broiler chicks. Asian J. Anim. Vet. Adv., 6: 80-87.

Imai, M., A. Ninomiya, H. Minekawa, T. Notomi, T. Ishizaki, M. Tashiro and T. Odagiri, 2006. Development of H5-RT-LAMP (loop-mediated isothermal amplification) system for rapid diagnosis of H5 avian influenza virus infection. Vaccine, 24: 6679-6682.

Lai, M.M. and D. Cavanagh, 1997. The molecular biology of coronaviruses. Adv. Virus. Res., 48: 1-100.

Mori, Y., K. Nagamine, N. Tomita and T. Notomi, 2001. Detection of loop-mediated isothermal amplification reaction by turbidity derived from magnesium pyrophosphate formation. Biochem. Biophys. Res. Commun., 289: 150-154.

Nagamine, K., T. Hase and T. Notomi, 2002. Accelerated reaction by loop-mediated isothermal amplification using loop primers. Mol. Cell. Probes, 16: 223-229. 
Notomi, T., H. Okayama, H. Masubuchi, T. Yonekawa, K. Watanabe, N. Amino and T. Hase, 2000. Loop-mediated isothermal amplification of DNA. Nucl. Acids Res., Vol. 28. 10.1093/nar/28.12. e63.

Pham, H.M., C. Nakajima, K. Ohashi and M. Onuma, 2005. Loop-mediated isothermal amplification for rapid detection of newcastle disease virus. J. Clin. Microbiol., 43: 1646-1650.
Swayne, D.E., 1998. A Laboratory Manual for the Isolation and Identification of Avian Pathogens. 4th Edn., American Association of Avian Pathologists, Kennett Square, PA., ISBN: 9780915538072 , Pages: 311.

Xue, C., Y. Zhang, Q. Zhou, C. Xu, X. Li and Y. Cao, 2009. Rapid detection of Infectious bursal Disease virus by reverse transcription loop-mediated isothermal amplification assay. J. Vet. Diagn. Invest., 21: $841-843$. 